\title{
Sensor Enhanced Health Information Systems: Issues and Challenges
}

\author{
https://doi.org/10.3991/ijim.v13i01.7037
}

\author{
Abubakar Adam, Adamu Abubakar ${ }^{(\varpi)}$, Murni Mahmud \\ International Islamic University Malaysia, Kuala Lumpur, Malaysia \\ adamu@i ium.edu.my
}

\begin{abstract}
Owing to the fact that the world population is aging and considering the high cost of healthcare services, researchers are exploiting the use of sensors to support mobile monitoring of healthcare systems remotely. However, this area is faced with numerous challenges. In this research, the challenges facing the integration of sensors in Health Information Systems (HIS) were reviewed. One of the major challenges faced by such systems is interoperability. In this article, a survey of existing mobile monitoring system is presented and the challenges facing this area are categorized. A significant contribution by some research has indicated the need for further study to thoroughly investigate Interoperability as a Property (IaaP) paradigm for HIS. Recommendation by some researchers requires that a prototype be developed in order to evaluate the performance of IaaP paradigm.
\end{abstract}

Keywords-Patient Monitoring Systems, Wireless Body Area Network, SmartShirt, M-Health, E-Health

\section{$1 \quad$ Introduction}

The world population is aging, as indicated in [1], and this lead to a situation whereby older people, critically ill, or disabled people will depend on younger ones for their daily needs. Unfortunately this dependency problem will get worse because the world population is aging [1]; population aging shows the changes in the proportion of different age groups usually based on a three-age-group population model: young age group $(<20)$, working age group $(20-64)$, and elderly people $(>64)[1]$. Based on population projection, the number of elderly people ( $>65$ years old) is expected to grow rapidly due to factors such as lack of fertility, mortality and migration. The trend in European Union (EU) population shows that while there is a decline in EU population, the population of people over the age of 65 will increase considerably. This trend is the same for any society because it is usually young adults that migrate to more developed countries for better life. This implies that elder people and kids that are below working class will be left behind. Similarly, when there is high mortality rate, due to catastrophic events such as war, it is usually young adults that are mostly affected, which also affects the population as a whole but that of the 
elderly is minimally affected and therefore while the population decreases that of the elder increases.

There is an indication that whereas in 2004 there was one elderly person ( $>65$ years old) for every four persons of 'working age' (20 to 64 years old), in 2050 there would be about one elderly person for every two of working age. A similar research was conducted by the UN and results show that; the proportion of older persons $(>60)$ in the world has been rising steadily, passing from 8 per cent in 1950 to 11 per cent in 2009 , and is expected to reach 22 per cent in 2050 . To add to the dependency problem, the elderly people are prone to diseases; the Berlin Aging Study (BASE) has shown that $88 \%$ of the persons aged above 70 years suffer from five or more somatic diseases. Hence, it is of utmost importance to exploit the opportunities provided by new information and communication technologies in order to support efficient health and social care, especially looking at the fact that health and social care systems services have high costs [1].

Due to population ageing, as described above, and the fact that healthcare is very expensive, technological advancements demands that different kinds of sensors be used to capture physiological signals from a patient's body who does not have to be physically in the hospital. The signals from the sensors will be sent to the health professionals, which can be used to monitor the patient remotely. In case of emergencies, health experts will prepare for the patients before reaching the hospital. Such monitoring systems will notify the health professionals about even the slightest change in the vital signals of the patient and therefore the patient can easily be monitored wherever he/she might be. Hence, older people, critically ill, and disabled people can achieve independent living with minimal human supervision. As a result, various technological aid are required, for example, a service-oriented architecture for wireless health systems has been presented [2]. Similarly, mHealth platform for Parkinson's disease patient management has been proposed, where sensors have been utilized to capture data and send to the cloud infrastructure to allow easy access for clinicians [3]. Sensors has been utilized to proposed a noncontact sleep monitoring system, in order to provide a sleeping status and breathing [4]. A concept based on correlating situation information and patient mood captured by sensors has been utilized for understanding mental health situation of individuals [5]. Temperature and pulse rate sensor and GSM technology has been used to proposed a medical sensing system that transfer information between a patient, doctor and the nearby paramedical unit [6]. Considering the above-mentioned studies, it is imperative to outline the challenges faced by the outpouring of such system. That is why this study, reviewed the existing challenges facing the available systems.

\section{Mobile Monitoring System of Healthcare}

The attempts to make health electronics started in the early 1950's. However, the use of mobile handheld devises to wirelessly access hospital data and the implementation of web-based e-health was not seen until mid-1990s. The use of hardware to support health-started way back in 1950's where only mainframes were 
used. Today handheld mobile devices are used for such purposes. ARPANET E-mail were used in 1970's for health related services while in the contemporary world 4G network are used [7].

\subsection{Architectural Description of the Existing Wireless-PDA based Systems}

A wireless-PDA based physiological monitoring system was presented in [8]. The system consists mainly of two parts: (i) the mobile unit, which is set up around the patient to acquire the patient's physiological data, and (ii) the management unit, which enables the medical staffs to telemonitor the patient's condition in real-time. Through the WLAN, the patient's biosignals can be transmitted in real-time to a remote central management unit, and authorized medical staff can access the data and the case history of the patient via the central management unit or the wireless devices. The management unit is usually a fixed computer within an existing hospital network or a mobile laptop via WLAN. However, Personal Digital Assistant (PDA) was used as a monitoring devise. PDA (such as Palm TX) is an old technology and consumed a lot of power. Furthermore, the mobile unit (patient environment), consultant terminal (doctors), and management unit (at the hospital) are expected to be within WLAN. If, for any reason, the car transporting the patient goes out of the network, the system will not work.

The Advanced Health and Disaster Aid Network (AID-N) system, which is sponsored by the National Library of Medicine was proposed in [9]. Three hierarchical layers are involved. The bottom layer consists of an ad hoc network of embedded medical systems that collect the vital signs of the patients. The second layer consists of servers that connect up to the backbone of the Internet to relay information to a central server on the third layer. The main benefits of the AID-N electronic triage include: (i) continuous monitoring of triage levels, physiological status, and location of the patients (ii) automated distribution of patient data in realtime to response team members both on and off the disaster site [9]. However, the framework is based PDA as the monitoring device, which is an old technology as stated earlier on. Moreover, for optimal performance, tags should not be more than one meter from the base station; which is clearly a limitation because dealing with patients of disaster might affect huge area.

\subsection{Architectural Description of the Existing Smartphone based Systems}

A typical example of a smartphone based WBAN healthcare structure for monitoring real time physiological parameters was presented in [10]. The WBAN consists of three types of modules: The first module-vital physiological parameters monitoring device-discusses the sensor technologies that can be used to read and monitor physiological parameters such as ECG, EEG, B. The second module Wireless Communication Standards for WBAN-deals with the wireless standards for transmitting the physiological data from the biosensors to the mobile phone. The third module-Software Development Phase for WBAN-has to do with software development for WBAN [10]. 
A low cost Windows Mobile BSN was proposed in [11]; one of the research objectives is to come up with some sort of correlation between core-body temperature and women fertility \& ovulation periods. Even though the work was centered on continuously measuring the body temperature, by inserting a sensor into a woman's vagina, it could also be extended to include other types of sensors. The proposed system consists of a SHIMMER-based body sensor network, which is used to measure and collect bio signals and send them to the processing unit using Bluetooth, which eventually send the signals to a mobile device also using Bluetooth. The system is consists of three modules (i) sensor (ii) processing unit and (iii) windows mobile device. However, only one sensor was used to measure cervix women's temperature and thus raise the issue of interoperability with other devices.

A personalized health monitoring system, using smart phones, is presented in [12]. The proposed system was designed for heart related diseases. The heart specialist can select one or more sensors to be used for a particular patient and configure the corresponding threshold levels for that patient. The application can generate alarms or warnings when thresholds have been reached. However, they identified in their research that power consumption still remains a challenge. A ubiquitous mobile health system for continuous monitoring of elderly patients from indoor and outdoor environments has been presented in [13]. The system consists of three components: (i) Wireless Wearable Body Area Network (WWBAN) (ii) Intelligent Central Node (ICN) (iii) Intelligent Central Server (ICS). The Intelligent Central Node (which is basically a smartphone) servers as an accelerometer, in the architecture, where it is responsible for collecting, and processing the data generated by the WWBAN (Wireless Wearable Body Area Network) sensors. After collecting all the sensor data, the ICN ensures that there is no change in the collected data before sending it to the Intelligent Central Server (ICS). Once the data is received by ICS, it is stored in the database for analysis. Ubiquitous mobile health monitoring system for elderly (UMHMSE), which is the prototype system, monitors location and health status through the use of Bluetooth and smartphone [13].

\subsection{Architectural Description of the Existing Location-based Systems}

A framework that extends the European Telecommunications Standards Institute (ETSI)/Parlay architecture, which is used for the deployment of standardized services over the next generation IP networks was proposed in [14]. Although, it is typically not a full-fledged location-based, but ETSI/Parlay architecture was expanded with new service capability features as well as sensor, profiling, and security mechanisms. In this architecture, NGeH domain is considered as an operational domain of welldefined state and transactions, where the patient interacts with various entities whose behavior is posed by constructed profiles. There are three types of peer entities that interact with each other: (i) subjects (e.g., doctors, nurses, pharmacists, family member) (ii) objects (ICT components that are deployed around the patient/subjects such as the biosensors, GPS, mobile terminals etc.) and (iii) operational domains (these are the places where the $\mathrm{NGeH}$ servers are provided such as hospitals, healthcare units, vehicles, homes etc.). In the new proposed framework architecture, 
the new set of SCFs (Service Capability Features) Interfaces is considered as extended set of SCFs Interfaces and the new Framework is considered as Extended Framework [14].

EmerLoc (Location-based services for emergency medical incidents) [15] and CAALYX (Complete Ambient Assisted Living Experiment) [16] are location-based systems for emergency purposes. Based on a middleware and network infrastructure, emergency incidents are handled in random locations either in a controlled environment (e.g. hospitals) or on sites where immediate health support is not possible (e.g., urban areas). Both EmerLoc and CAALYX incorporates wearable devices comprising of wireless sensors that continuously monitor the user's biosignals, a micro computing unit, which is responsible for processing sensor readings and a central monitoring unit (CMU), which transmits the information to the medical personnel. Patient's device (PD) sends the patient's bio signals and data about the current location of the patient to the Central Monitoring Unit (CMU). Therefore, if there is any critical situation the PD (first level of incident handling) will first detect it and then it will be communicated to the CMU (second level of incident handling). The CMU is capable of alerting the attending doctor (or any doctor in proximity to the patient) on the situation and advise him to handle the incident. However, one of the major problems of this architecture is that it involves the use of heterogeneous network infrastructures (e.g., WLAN, PAN, GSM/UMTS) and the exploitation of the position fixing technologies that such networks offer. CAALYX has three main areas of development (i) Roaming Monitoring System (RMS), which monitors the older person independent of where he is at home or outdoors (ii) The Home Monitoring System (HMS), which is intended to integrate other home devices in order to improve health services that can be provide to the patient and (iii) The Central Care Service and Monitoring System (CCS/MS) is intended to serve as the control center where alerts will be received and determine the necessary cause of action.

\subsection{Architectural Description of the Existing Wearable-based Systems}

The use of wearable smart shirt for healthcare was proposed in [17], which measures electrocardiogram (ECG) and acceleration signals for continuous and real time health monitoring. The architecture consists of multi-hop sensors in smart shirt having Universal Serial Bus (USB) programming board as a separate module. The size of integrated wearable sensor nodes is reduced by mounting the device on two process control blocks. The smart shirt, hence, consists of a wireless sensor node, an ECG and accelerometer sensor board and conductive fabric electrodes for ECG measurement in normal shirt design. Similarly, an approach to monitor Blood Pressure (BP) cufflessly and continuously using health-Shirt (h-Shirt) was proposed in [18]. The h-Shirt is designed to record ECG and photoplethysmogram (PPG) at the same time and to use them to estimate BP with the PTT-based approach. ECG is captured from the two wrists, with reference to an electrode placed on the forearm to avoid noise induced by respiration. Recent development of Wearable Intelligent Systems for e-Health (WISEs) is reviewed in [19], including breakthrough technologies and technical challenges that remain to be solved. 


\subsection{Issues with the Architectural Framework of the Existing Systems}

One of the major issue of wearable sensor devices, like the ones on smart shirt, is that the shirt should be non-invasive in such a way that the patient can carry out his/her daily activities with ease. In addition, interference from the fabric of the shirt could also cause a challenge.

All the architectures/frameworks presented so far are designed by integrating specific sensors, which can send data to a processing unit using some communication protocols such as Bluetooth, WLAN etc. For the most part, in their design, they are standalone and hence cannot fully support the interoperability functions, which are highly required in such systems. Some commercial solutions to integrate devices compliant with the HDP, such as the Bluegiga AP3201 e-Health Gateway are available in the market. However, the interoperability is limited to only HDP devices and therefore does not satisfy the requirement of integrating devices heterogeneously [20].

The Internet could provide the pillars upon which solutions to such problems could be achieved where it is used as a medium to integrate clinical devices in a term called the Future Internet of Things (IoT). Internet of Things is considered as one of the major communication advances in recent years because it offers the basis for the development of independent cooperative services and applications. Assisted Living Environments for patients with respiratory illnesses under the AIRE project was presented in [20]. Monere and Movital are hardware resources, which make heterogeneous devices to communicate smoothly. The solution is designed to serve as a bridge between the sensors connected to the patient and the information systems so that a Ubiquitous Integration of Clinical Environments can be achieved. With respect to hardware, the framework offers a gateway and a personal clinical device used for the wireless transmission of continuous vital signs through 6LoWPAN, and patient identification through RFID. While in terms of software, the framework presented YOAPY, which is a protocol that attempts to provide an efficient, secure, and scalable integration of the sensors deployed in the patient's personal environment [20]. However, owing to the fact that IoT consist of increasingly heterogeneous set of devices that exchange relevant information, interoperability remains an issue. The proposed architecture may face problems later when new communication protocols and standards are introduced to the fast growing world of information technology.

To address the problem of interoperability, a concept of interoperability as a property of a single system/devise rather than in connection to other pre-committed systems was presented in [21]. The research subsequently attempted to assess and exemplify the impact that Interoperability as a Property (IaaP) paradigm on the healthcare information systems' landscape. Sensors are attached or implanted into patient's body, which will be used to measure physiological data from the patient's body and this form what is known as Body Area Network (BAN). In the patient's environment, there might be some sensors that measure environmental signals, which might be useful for data analysis. The body sensors and the environmental plus the processing unit make up what is known as Personal Area Network (PAN). All sensory ata are first transmitted to a local processing unit using some network/communication 
medium. The processing unit can be a PDA, smart phone or other complex sensors, which can be used for pre-processing before sending the data to a Health Information System.

\section{Challenges of Using Sensor(S) Networks for Healthcare}

The major challenges observed for using sensor(s) networks for healthcare are categorized into four, namely: (i) Challenges based on OSI layers/level (ii) Challenges based on network types (iii) Challenges based on data processing and interpretation (iv) Challenges Based on the Category of the Challenges. It is worth mentioning here that these challenges affect, mobile monitoring systems, irrespective of the target group (elderly, critically ill etc.)

\subsection{Challenges Based on OSI Layer/Level}

The application of sensors in healthcare has a lot of benefits. However, there are numerous challenges of wireless sensors when used in the healthcare. Such challenges can be viewed from different perspectives. Table 1 and Table 2 and are based on the survey presented in [23 and 24] and they present the challenges of using sensor networks for health care with respect to OSI layers and network types respectively.

Table 1. Challenges based on OSI Layers

\begin{tabular}{|c|c|c|}
\hline Level/Layer & Challenge & Discussion \\
\hline \multirow{4}{*}{ Hardware Level } & Unobtrusiveness & $\begin{array}{l}\text { Integrating many sensors to be used by one patient makes } \\
\text { it even more difficult for the system/architectures to be as } \\
\text { unobtrusive as possible. }\end{array}$ \\
\hline & $\begin{array}{l}\text { Sensitivity and } \\
\text { calibration }\end{array}$ & $\begin{array}{l}\text { Sensitivity of the sensors in accurate measuring data from } \\
\text { the patient body especially in different operational } \\
\text { conditions; such as raining condition, exercising } \\
\text { condition etc. }\end{array}$ \\
\hline & Energy & $\begin{array}{l}\text { Energy consumption, by the sensors, is one of the major } \\
\text { challenges. }\end{array}$ \\
\hline & $\begin{array}{l}\text { Data Acquisition } \\
\text { Efficiency }\end{array}$ & $\begin{array}{l}\text { The rate at which the sensors collect data is also a } \\
\text { challenge at the hardware level. }\end{array}$ \\
\hline \multirow{3}{*}{ Physical Layer } & $\begin{array}{l}\text { Error resilience and } \\
\text { reliability }\end{array}$ & $\begin{array}{l}\text { Quite a number of sensors are affected by Signal-to-Noise } \\
\text { Ratio (SNR) hence causing higher bit error rates and } \\
\text { thereby reducing the reliable coverage area. }\end{array}$ \\
\hline & Interoperability & $\begin{array}{l}\text { The fact that many sensors might be connected together } \\
\text { and each might be operating at different frequencies raise } \\
\text { the problem of interoperability. }\end{array}$ \\
\hline & Bandwidth & $\begin{array}{l}\text { The bandwidth available for data communication for } \\
\text { wireless body area networks is relatively low thereby } \\
\text { affecting the performance of the whole system. }\end{array}$ \\
\hline MAC Layer & \multicolumn{2}{|c|}{$\begin{array}{l}\text { Some challenges are addressed at the MAC level such as the Quality of Service } \\
\text { requirements of emergency traffic. }\end{array}$} \\
\hline Network Layer & \multicolumn{2}{|c|}{$\begin{array}{l}\text { Due to the huge amount of traffic that are common in wireless sensor networks } \\
\text { may cause data movement difficulty at the nodes closer to the base station. }\end{array}$} \\
\hline Transport Layer & \multicolumn{2}{|c|}{$\begin{array}{l}\text { It is of utmost importance to have a congestion and flow control mechanisms at } \\
\text { the transport layer. }\end{array}$} \\
\hline Application Layer & \multicolumn{2}{|c|}{$\begin{array}{l}\text { Challenges at the application layer has to do with how the data is organized, } \\
\text { analyzed in such a way that meaningful information can derived to help in }\end{array}$} \\
\hline
\end{tabular}




\begin{tabular}{|c|c|c|}
\hline Level/Layer & Challenge & Discussion \\
\hline & decision making. & \\
\hline \multirow{5}{*}{ Layer Independent } & Security & \multirow{5}{*}{$\begin{array}{l}\text { As far as the OSI Layer is concerned, the challenges } \\
\text { identified here are not directly related to any layer; but } \\
\text { are of utmost importance to be considered when dealing } \\
\text { with sensors in healthcare related systems. }\end{array}$} \\
\hline & Privacy & \\
\hline & User-friendliness & \\
\hline & $\begin{array}{l}\text { Ease of deployment } \\
\text { and scalability }\end{array}$ & \\
\hline & Mobility & \\
\hline
\end{tabular}

\subsection{Challenges Based on Network Types}

Pervasive healthcare monitoring systems dwells on five subsystems [23-34] as discussed in Table 2, which enumerates the types of subsystem or network alongside challenges that are commonly faced by such system or networks. The table further discusses each type of network with respect to the design consideration.

Table 2. Challenges based on subsystems/network types

\begin{tabular}{|c|c|c|}
\hline Subsystem/Network & Design Consideration & Discussion \\
\hline $\begin{array}{l}\text { Medical Body Area } \\
\text { Network (MBAN) }\end{array}$ & $\begin{array}{l}\text { Power consumption } \\
\text { Transmission } \\
\text { Power Unobtrusiveness } \\
\text { Portability } \\
\text { Real-time availability } \\
\text { Reliable communications } \\
\text { Multi-hop routing } \\
\text { Security/Localization of } \\
\text { micro-robot inside the body }\end{array}$ & $\begin{array}{l}\text { As the name implies, A Medical Body } \\
\text { Area Network (MBAN) is a network } \\
\text { that is built around the human body. } \\
\text { Usually it consists of wireless devices } \\
\text { that are either (i) implanted within the } \\
\text { human body or (ii) attached to the } \\
\text { human body in a form of wrist-band, e- } \\
\text { shirt etc. All the challenges identified } \\
\text { here, directly or indirectly affect the } \\
\text { performance of the Wireless Body Area } \\
\text { Network. }\end{array}$ \\
\hline $\begin{array}{l}\text { Wireless Personal Area } \\
\text { Network (WPAN) }\end{array}$ & $\begin{array}{l}\text { Energy efficiency } \\
\text { Scalability } \\
\text { Self-organization between } \\
\text { the nodes }\end{array}$ & $\begin{array}{l}\text { A WPAN is a low range wireless } \\
\text { network that consists of personal } \\
\text { devices of an individual such as } \\
\text { computers, cellphones, and personal } \\
\text { digital assistant. It uses technology that } \\
\text { can be used to exchange data over short } \\
\text { distance such as Bluetooth, NFC, } \\
\text { ZigBee, IrDA (Infrared Data } \\
\text { Association). Scalability, self- } \\
\text { organization between nodes and other } \\
\text { similar challenges need to be properly } \\
\text { addressed in this subsystem. }\end{array}$ \\
\hline $\begin{array}{l}\text { Gateway to the Wide Area } \\
\text { Networks }\end{array}$ & $\begin{array}{l}\text { Security Congestion } \\
\text { prevention }\end{array}$ & $\begin{array}{l}\text { Typically, a WAN gateway is a network } \\
\text { device such as network switch, a } \\
\text { wireless router, a wireless access point } \\
\text { etc.; that allows the connection of the } \\
\text { WAN with other networks. Due to the } \\
\text { high level of data traffic passing } \\
\text { through this point security and data } \\
\text { congestion are issues that needs to be } \\
\text { properly looked into at this point. }\end{array}$ \\
\hline Wide Area Networks & $\begin{array}{l}\text { Data rate, Reliable } \\
\text { communication protocols, } \\
\text { Secure data transmission, } \\
\text { Coverage }\end{array}$ & $\begin{array}{l}\text { All the challenges mentioned here } \\
\text { should be carefully addressed at this } \\
\text { level. }\end{array}$ \\
\hline $\begin{array}{l}\text { End-user healthcare } \\
\text { monitoring application }\end{array}$ & $\begin{array}{l}\text { Privacy, Security Reliability } \\
\text { User-friendliness, }\end{array}$ & $\begin{array}{l}\text { All the challenges mentioned here } \\
\text { should be carefully addressed at this }\end{array}$ \\
\hline
\end{tabular}




\begin{tabular}{|l|l|l|}
\hline Subsystem/Network & \multicolumn{1}{|c|}{ Design Consideration } & \multicolumn{1}{c|}{ Discussion } \\
\hline & Middleware design & level. \\
& $\begin{array}{l}\text { Scalability, Interoperability } \\
\text { Context-awareness }\end{array}$ & \\
\hline
\end{tabular}

\subsection{Challenges Based on Data Processing and Interpretation}

With the huge amount of data collected from wearable sensors used for physiological monitoring, it is difficult to expeditiously processed and interpret such data. Therefore, we are faced with numerous challenges, when analyzing and interpreting data from wearable wireless sensor networks. Data mining related challenges are discussed in [35-38]:

- Need for Large scale monitoring in non-clinical context

- Dealing with annotated data sets

- Multiple measurements

- Contextual information

- Reliability, level of trust to the system

- Discovering of unseen features

- Post-processing and Representation

\subsection{Challenges Based on the Category of the Challenges}

Another angle from which the challenges can be viewed are discussed in the Table 3.

Table 3. Challenges based on their natures

\begin{tabular}{|l|l|l|}
\hline \multicolumn{1}{|c|}{ Subsystem/Network } & Design Consideration & \multicolumn{1}{c|}{ Discussion } \\
\hline Security related & $\begin{array}{l}\text { Security } \\
\text { Privacy }\end{array}$ & $\begin{array}{l}\text { Looking at the tendency of processing } \\
\text { huge amount of data, privacy and security } \\
\text { is always an issue. }\end{array}$ \\
\hline Communication related & $\begin{array}{l}\text { Bandwidth } \\
\text { Data acquisition } \\
\text { Interoperability }\end{array}$ & $\begin{array}{l}\text { The challenges here have to do with } \\
\text { communication related issues. }\end{array}$ \\
\hline User Acceptance related & $\begin{array}{l}\text { Unobtrusiveness } \\
\text { Mobility } \\
\text { User friendliness }\end{array}$ & $\begin{array}{l}\text { The challenges here have to do more with } \\
\text { the willingness, comfort and the ease with } \\
\text { which the user interact with the systems. }\end{array}$ \\
\hline Functional related & $\begin{array}{l}\text { Energy consummation, } \\
\text { Interoperability } \\
\text { Error Resilience, } \\
\text { Reliability, Ease of } \\
\text { deployment, } \\
\text { Sensitivity/calibration }\end{array}$ & $\begin{array}{l}\text { The challenges here have to do with the } \\
\text { actual function of the devise itself. } \\
\text { Therefore, they are more related to the } \\
\text { sensors themselves. }\end{array}$ \\
\hline
\end{tabular}

\section{$4 \quad$ Interoperability as a Property (IaaP)}

Sensor is very important, that is why is used for healthcare, Vibration sensor is used in fault detection of solenoid valves by the signal gathered in Labview SignalExpress system [39]. A sensor based framework for real-time sensing of the 
state of equipment of post-prognostics decision has been proposed. This can help in understanding malfunction or failure in the system [40]. Wireless body area network has been utilized to proposed a model for patient that requires continues monitoring, through the use of the remote monitoring application [41]. Remote and continuous monitoring of individual's health technique using wearable motion sensor has been proposed through integrating body activity and sensor location [42-44]. With the exponentially increasing number of devices that are connected to what is termed as the Internet of Things, it is extremely difficult for devices to interoperate. Currently, devices interoperate based on pre-agreements of the various devices to exchange information and to act upon this information. The major problem with these arrangements is that they lead to application silos with fragmented architectures, which will lead to potentially biased observations. For example diastolic blood pressure is affected by environmental temperature [21].Therefore, diastolic blood pressure could be wrongly observed if environmental temperature is not considered and this can lead to wrong decision. Hence, if many sensors are to work together accordingly then the following scenario should be in place. Consider a setup with three sensors $\mathrm{P}, \mathrm{Q}$ and R. Observations by $\mathrm{P}, \mathrm{Q}$ and $\mathrm{R}$ can be represented using OP, $\mathrm{OQ}$ and $\mathrm{OR}$ respectively; and messages sent by $\mathrm{P}, \mathrm{Q}$ and $\mathrm{R}$ can be represented using MP, MQ and MR respectively as shown in the following diagram.

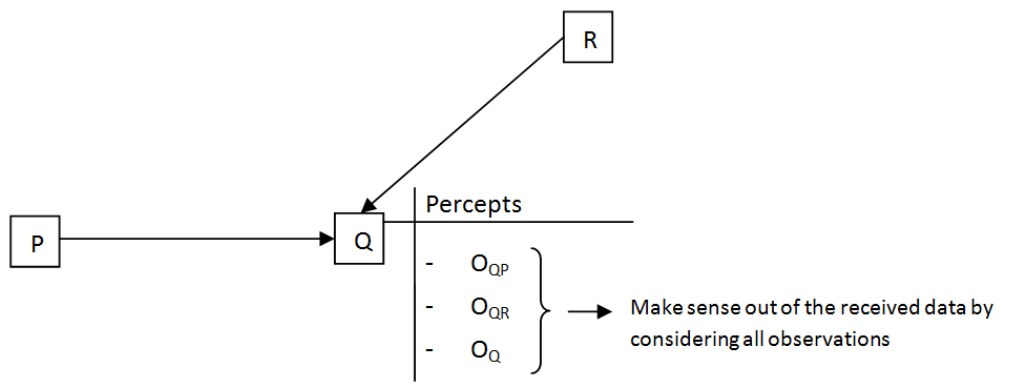

Fig. 1. IaaP Concepts

In the environment shown in Figure1, $\mathrm{P}$ sends a message (MP) to $\mathrm{Q}$, where $\mathrm{Q}$ observes the message from $\mathrm{P}$ and can be represented as OQP. Similarly, R sends a message (MR) to $\mathrm{Q}$, where $\mathrm{Q}$ observes the message from $\mathrm{R}$ and can be represented as OQR. At the same time, $\mathrm{Q}$ is making its own observation as OQ. When all these observations are put together as percepts then a meaningful decision can be taken else any decision taken without considering all the observations in may lead to wrong conclusions. Since, there could be many sensors in the environment, it is important the $\mathrm{Q}$ makes intelligent decision.

\subsection{Challenges Based on Data Processing and Interpretation}

In the current Wireless Sensor Network (WSN) setting, for two or more devices/components to communicate they must agree on the standard and format to 
interoperate. With the increasing number of devise that are connected to the network in what is termed as IoT, devices should be able to identify and communicate with devices on the network without any prior agreements. This can be achieved if each "thing" on the network exhibits certain attributes/factors.

The enabling factors for IaaP are [21]: (i) Awareness: has two aspects: selfawareness and environmental awareness. As the name implies, self-awareness is the ability of the system to be aware of events within itself such as available energy levels. On the other hand, environmental awareness is related to the capability of the system to sense events from its environment. (ii) Perceptivity: is the capability of the system to assign a meaning to an observation. (iii) Intelligence: encompasses assertion, storing and acquisition of the behavior patterns in order to take intelligent decisions for actions to be taken. (iv) Extroversion: is related to the willingness and capability of the system to articulate its actions.

\subsection{Challenges Based on Data Processing and Interpretation}

Due to technical reasons, power related issues, and mobility, systems/subsystems could be disconnected and reconnected to a network. Hence, in addition to the above four enabling factors identified in [21], two more factors should be considered. These factors are: (i) Track and (ii) Trace.

Track: Locate where objects are; it is important to locate where objects are in order to make intelligent decisions.

Trace: Ability, of the "thing", to log its sensor measurements so that when it is outside a network all readings can be log. The moment it gets reconnected to a network all the readings (in the history that was logged) can be downloaded or and communicated to other "things" if needed.

Refined Setup: Sensors in IaaP enabled environment can be classified into two: (i) Master sensor (ii) Auxiliary (supporting or primary) sensor. The function of each type of sensor is shown in Figure 2.

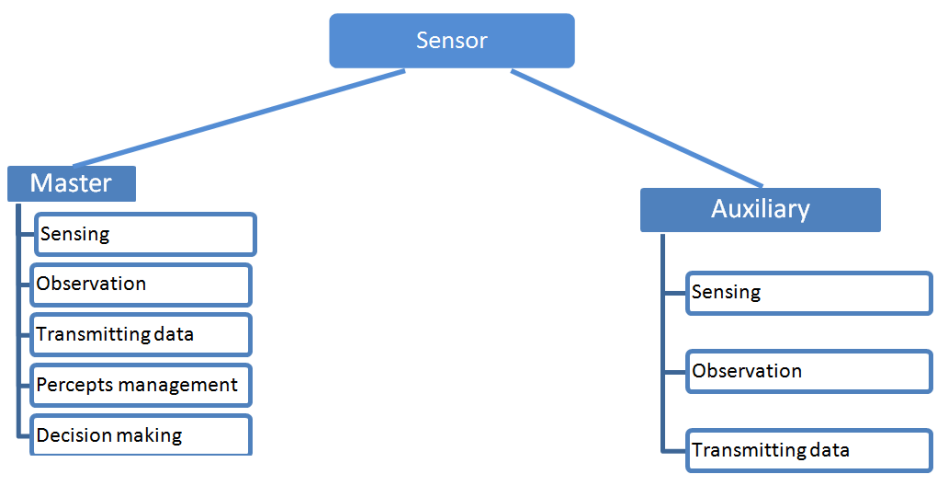

Fig. 2. Sensor classification based on IaaP Concepts 
While the auxiliary sensor is designed to be used in measuring basic sensory data (such as temperature, pressure etc.) the master sensor is designed to have some processing capability. In addition to sensing and transmitting data, the Master sensor should have the capability of collecting and managing percepts in order to make sense out of the numerous observations received from auxiliary sensors; and subsequently making intelligent decision. Therefore, an IaaP enabled environment can be represented using the diagram shown in Figure 3.

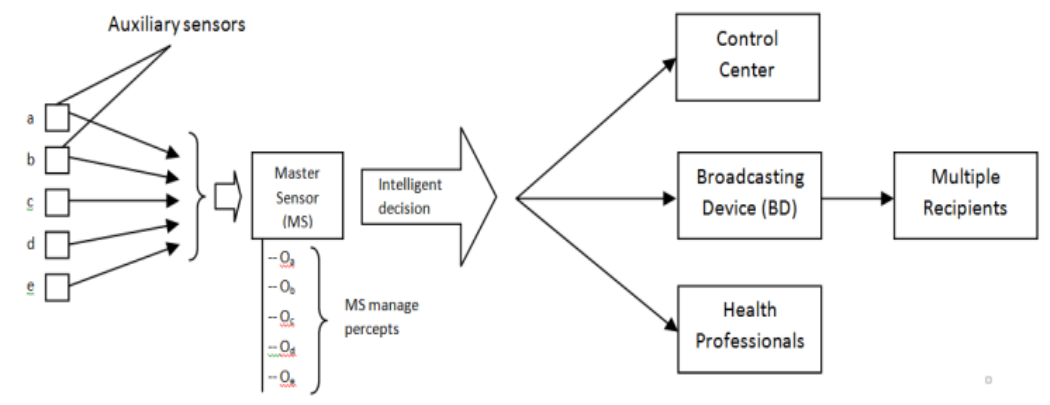

Fig. 3. Representation of IaaP enabled environment

As indicated in the diagram above, the auxiliary sensors measure individual senor data and transmit it to the master sensor, which has the capability of managing percepts in order to make sense out of all the collected observations. Based on all the operations, MS makes an intelligent decision on what to do (for example call the medical doctor) and sends it directly to the health professional (such as the medical doctor), control center or to a broadcasting device which will in turn broadcast it to multiple recipients which include loved ones, health professionals, ambulance department etc.

\section{Conclusion}

A survey of the different approaches for proposing patient monitoring system was presented. There are three major components required in building Sensor Enhanced HIS. Previous studies have identified them to be: (i) Network technologies (ii) Sensor $\&$ actuators and (iii) Processing Unit. Numerous challenges are faced when developing such systems. Such challenges affect the performance, integrity and acceptability of Sensor-Enhanced HIS. The challenges affecting the integration of sensors in Health Information System (HIS) are categorized. Interoperability as a Property (IaaP) paradigm has been found to be a promising approach that could unravel the problems of interoperability among interacting systems, subsystems or devises. IaaP is a promising approach that will go a long way to solve quite a number of the problems faced by elderly, critically ill or young children who depend on others for their daily need. Thus, thorough studies are required on refining the enabling 
factors as well as intensive implementation of prototype to further revamp the new paradigm.

\section{Acknowledgement}

This paper was supported by a Research Initiative Grant Scheme (No. RIGS16364-0528) from the International Islamic University of Malaysia (IIUM).

\section{$7 \quad$ References}

[1] Koch, S., Marschollek, M., Wolf, K. H., Plischke, M., \& Haux, R. (2009). On healthenabling and ambient-assistive technologies. Methods Inf Med, 48(1), 29-37. https://doi.org/10.3414/ME9136

[2] Balampanis, S., Sotiriadis, S., \& Petrakis, E. G. (2016). Internet of Things Architecture for Enhanced Living Environments. IEEE Cloud Computing, 3(6), 28-34. https://doi.org/1 0.1109/MCC.2016.128

[3] Tsiouris, K. M., Gatsios, D., Rigas, G., Miljkovic, D., Seljak, B. K., Bohanec, M., \& Fotiadis, D. I. (2017). PD_Manager: an mHealth platform for Parkinson's disease patient management. Healthcare Technology Letters. https://doi.org/10.1049/ht1.2017.0007

[4] Lin, F., Zhuang, Y., Song, C., Wang, A., Li, Y., Gu, C., \& Xu, W. (2017). SleepSense: A Noncontact and Cost-Effective Sleep Monitoring System. IEEE transactions on biomedical circuits and systems, 11(1), 189-202. https://doi.org/10.1109/TBCAS.2016.2541680

[5] Teles, A. S., Rocha, A., Silva, F. J., Lopes, J. C., OSullivan, D., Van de Ven, P., \& Endler, M. (2016). Towards Situation-aware Mobile Applications in Mental Health. In ComputerBased Medical Systems (CBMS), 2016 IEEE 29th International Symposium on (pp. 349354). https://doi.org/10.1109/CBMS.2016.7

[6] Suganya, M., \& Jayanthy, S. (2016,). Telehealth system for home environment. In Emerging Technological Trends (ICETT), International Conference on (pp. 1-6). https://doi.org/10.1109/ICETT.2016.7873678

[7] Pawar, P., Jones, V., Van Beijnum, B. J. F., \& Hermens, H. (2012). A framework for the comparison of mobile patient monitoring systems. Journal of biomedical informatics, 45(3), 544-556. https://doi.org/10.1016/j.jbi.2012.02.007

[8] Lin, Y. H., Jan, I. C., Ko, P. I., Chen, Y. Y., Wong, J. M., \& Jan, G. J. (2004). A wireless PDA-based physiological monitoring system for patient transport. IEEE Transactions on information technology in biomedicine, 8(4), 439-447. https://doi.org/10.1109/ TITB.2004.837829

[9] Gao, T., Massey, T., Selavo, L., Crawford, D., Chen, B. R., Lorincz, K., \& Chanmugam, A. (2007). The advanced health and disaster aid network: A light-weight wireless medical system for triage. IEEE Transactions on biomedical circuits and systems, 1(3), 203-216. https://doi.org/10.1109/TBCAS.2007.910901

[10] Singh, M., \& Jain, N. (2014). A survey on integrated wireless healthcare framework for continuous physiological monitoring. International Journal of Computer Applications, 86(13). https://doi.org/10.5120/15048-3416

[11] Pereira, O. R., Caldeira, J. M., Shu, L., \& Rodrigues, J. J. (2014). An efficient and low cost Windows Mobile BSN monitoring system based on TinyOS. Telecommunication Systems, 55(1), 115-124. https://doi.org/10.1007/s11235-013-9756-4 
[12] Gay, V., \& Leijdekkers, P. (2007). A health monitoring system using smart phones and wearable sensors. International journal of ARM, 8(2), 29-35.

[13] Bourouis, A., Feham, M., \& Bouchachia, A. (2011) "Ubiquitous Mobile Health Monitoring System for Elderly (UMHMSE)." International Journal of Computer Science and Information Technology (IJCSIT), Vol. 3 No. 3, pp. 74-82 2011. https://doi.org/10.5121/ijcsit.2011.3306

[14] Fengou, M. A., Mantas, G., Lymberopoulos, D., Komninos, N., Fengos, S., \& Lazarou, N. (2013). A new framework architecture for next generation e-health services. IEEE journal of biomedical and health informatics, 17(1), 9-18. https://doi.org/10.1109/ TITB.2012.2224876

[15] Maglogiannis, I., \& Hadjiefthymiades, S. (2007). EmerLoc: Location-based services for emergency medical incidents. International journal of medical informatics, 76(10), 747759. https://doi.org/10.1016/j.ijmedinf.2006.07.010

[16] Boulos, M. N. K., Rocha, A., Martins, A., Vicente, M. E., Bolz, A., Feld, R., \& Sdogati, C. (2007). CAALYX: a new generation of location-based services in healthcare. International journal of health geographics, 6(1), 9. https://doi.org/10.1186/1476-072X-6-9

[17] Lee, Y. D., \& Chung, W. Y. (2009). Wireless sensor network based wearable smart shirt for ubiquitous health and activity monitoring. Sensors and Actuators B: Chemical, 140(2), 390-395. https://doi.org/10.1016/j.snb.2009.04.040

[18] Zhang, Y. T., Poon, C. C., Chan, C. H., Tsang, M. W., \& Wu, K. F. (2006). A health-shirt using e-textile material for the continuous and cuffless monitoring of arterial blood pressure. In Medical Devices and Biosensors, 2006. 3rd IEEE/EMBS International Summer School on (pp. 86-89). IEEE. https://doi.org/10.1109/ISSMDBS.2006.360104

[19] Poon, C. C., Liu, Q., Gao, H., Lin, W. H., \& Zhang, Y. T. (2011). Wearable intelligent systems for e-health. Journal of Computing Science and Engineering, 5(3), 246-256. https://doi.org/10.5626/JCSE.2011.5.3.246

[20] Jara, A. J., Zamora-Izquierdo, M. A., \& Skarmeta, A. F. (2013). Interconnection framework for mHealth and remote monitoring based on the internet of things. IEEE Journal on Selected Areas in Communications, 31(9), 47-65. https://doi.org/10.1109/JSAC 2013.SUP.0513005

[21] Zdravković, M., Noran, O., Trajanović, M. (2014). On Pervasive Healthcare Information Systems in the Internet of Things." 25th Australasian conference on Information System (ACIS2014), Auckland, NZ; 2014.

[22] Paliwal, G., \& Kiwelekar, A. W. (2013, March). A comparison of mobile patient monitoring systems. In International Conference on Health Information Science (pp. 198209). Springer, Berlin, Heidelberg. https://doi.org/10.1007/978-3-642-37899-7_17

[23] Jones, V., van Halteren, A., Dokovsky, N., Koprinkov, G., Bults, R., Konstantas, D., \& Herzog, R. (2006). Mobihealth: Mobile health services based on body area networks. In M-Health (pp. 219-236). Springer US. https://doi.org/10.1007/0-387-26559-7_16

[24] V.Halteren et al. "Mobile patient monitoring: the MobiHealth system" J InfTechnolHealthc 2004; 2(5):365-73.

[25] Jones, V., Gay, V., \& Leijdekkers, P. (2010). Body sensor networks for mobile health monitoring: Experience in europe and australia. In Digital Society, 2010. ICDS'10. Fourth International Conference on (pp. 204-209). IEEE. https://doi.org/10.1109/ICDS.2010.41

[26] Jezewski, J., Pawlak, A., Wróbel, J., Horoba, K., \& Penkala, P. (2015). Towards a medical cyber-physical system for home telecare of high-risk pregnancy. IFAC-PapersOnLine, 48(4), 466-473. https://doi.org/10.1016/j.ifacol.2015.07.079 
[27] Bott, O. J., Marschollek, M., Wolf, K. H., \& Haux, R. (2007). Towards new scopes: sensor-enhanced regional health information systems-part 1: architectural challenges. Methods of information in medicine, 46(4), 476-483. https://doi.org/10.1160/ME9057

[28] Khan, F. A., Ali, A., Abbas, H., \& Haldar, N. A. H. (2014). A cloud-based healthcare framework for security and patients' data privacy using wireless body area networks. Procedia Computer Science, 34, 511-517. https://doi.org/10.1016/j.procs.2014.07.058

[29] Angood, P. B., Satava, R., Doarn, C., \& Merrell, R. (2000). Telemedicine at the top of the world: the 1998 and 1999 Everest extreme expeditions. Telemedicine Journal and eHealth, 6(3), 315-325. https://doi.org/10.1089/153056200750040174

[30] Gao, T., Massey, T., Selavo, L., Crawford, D., Chen, B. R., Lorincz, K., \& Chanmugam, A. (2007). The advanced health and disaster aid network: A lightweight wireless medical system for triage. IEEE Transactions on biomedical circuits and systems, 1(3), 203-216. https://doi.org/10.1109/TBCAS.2007.910901

[31] Gay, V., \& Leijdekkers, P. (2007). A health monitoring system using smart phones and wearable sensors. International journal of ARM, 8(2), 29-35.

[32] Lin, Y. H., Jan, I. C., Ko, P. I., Chen, Y. Y., Wong, J. M., \& Jan, G. J. (2004). A wireless PDA-based physiological monitoring system for patient transport. IEEE Transactions on information technology in biomedicine, 8(4), 439-447. https://doi.org/10.1109/TI TB.2004.837829

[33] Wai, A. A. P., Fook, V. F. S., Jayachandran, M., Biswas, J., Nugent, C., Mulvenna, M., \& Kiat, P. Y. L. (2008). Smart wireless continence management system for persons with dementia. Telemedicine and e-Health, 14(8), 825-832. https://doi.org/10. $1089 / \mathrm{tmj} .2008 .0084$

[34] Alemdar, H., \& Ersoy, C. (2010). Wireless sensor networks for healthcare: A survey. Computer Networks, 54(15), 2688-2710. https://doi.org/10.1016/j.comnet.2010.05.003

[35] Zorzi, M., Gluhak, A., Lange, S., \& Bassi, A. (2010). From today's intranet of things to a future internet of things: a wireless-and mobility-related view. IEEE Wireless Communications, 17(6). https://doi.org/10.1109/MWC.2010.5675777

[36] Atzori, L., Iera, A., \& Morabito, G. (2010). The internet of things: A survey. Computer networks, 54(15), 2787-2805. https://doi.org/10.1016/j.comnet.2010.05.010

[37] Istepanian, R. S., Hu, S., Philip, N. Y., \& Sungoor, A. (2011). The potential of Internet of $\mathrm{m}$-health Things " $\mathrm{m}$-IoT" for non-invasive glucose level sensing. In Engineering in Medicine and Biology Society, EMBC, 2011 Annual International Conference of the IEEE (pp. 5264-5266). IEEE. https://doi.org/10.1109/IEMBS.2011.6091302

[38] Banaee, H., Ahmed, M. U., \& Loutfi, A. (2013). Data mining for wearable sensors in health monitoring systems: a review of recent trends and challenges. Sensors, 13(12), 17472-17500. https://doi.org/10.3390/s131217472

[39] Guo, H., Wang, K., Cui, H., Xu, A., \& Jiang, J. (2016). A Novel Method of Fault Detection for Solenoid Valves Based on Vibration Signal Measurement. In Internet of Things (iThings) and IEEE Green Computing and Communications (GreenCom) and IEEE Cyber, Physical and Social Computing (CPSCom) and IEEE Smart Data (SmartData), 2016 IEEE International Conference on (pp. 870-873). IEEE. https://doi.org/10.1109/iThings-GreenCom-CPSCom-SmartData.2016.179

[40] Meraghni, S., Terrissa, L. S., Zerhouni, N., Varnier, C., \& Ayad, S. (2016). A postprognostics decision framework for cell site using Cloud computing and Internet of Things. In Cloud Computing Technologies and Applications (CloudTech), 2016 2nd International Conference on (pp. 310-315). IEEE. https://doi.org/10.1109 /CloudTech.2016.7847715 
[41] Nubenthan, S., \& Shalomy, C. (2017). A wireless continuous patient monitoring system for dengue: Wi-Mon. In Technology and Management (NCTM), National Conference on (pp. 23-27). IEEE.

[42] Mortazavi, B., Pourhomayoun, M., Ghasemzadeh, H., Jafari, R., Roberts, C. K., \& Sarrafzadeh, M. (2015). Context-aware data processing to enhance quality of measurements in wireless health systems: An application to met calculation of exergaming actions. IEEE Internet of Things Journal, 2(1), 84-93. https://doi.org/10. 1109/JIOT.2014.2364407

[43] De Castro Oliveira, I. F. (2012). A telematic platform towards regional connected healthcare (Doctoral dissertation, Universidade de Aveiro (Portugal))

[44] Hsu, I. C. (2013). Wireless Context-Aware Healthcare System Based on Sensor Web 2.0. International Journal of Innovation, Management and Technology, 4(4), 414. https://doi.org/10.7763/IJIMT.2013.V4.432

\section{Authors}

Abubakar Adam is a PhD student in the Department of Computer Science at the International Islamic University of Malaysia. His research interests include software matrix, Internet of things and sensor networks.

Murni Mahmud is an Associate Professor with the Department of Information System at the International Islamic University of Malaysia. Her current research interest is in the area of Human Computer Interaction.

Adamu Abubakar is an Assistant Professor with the Department of Computer Science at the International Islamic University of Malaysia. His current research interests include machine learning and computer networking.

Article submitted 24 April 2017. Resubmitted 23 September 2017 and 23 August 2018. Final acceptance 14 December 2018. Final version published as submitted by the authors. 